\title{
INSTRUMENTOS DE EVALUACIÓN DEL DESARROLLO MOTOR
}

Recibido 24-IX-2001

Resumen: La evaluación de nuestros niños preescolares y durante el primer ciclo en el campo del desarrollo motor ha generado cuestionamientos importantes en los últimos años. Ya que se concibe como elemento de educación formal y como auspiciador de conocimiento de la integralidad del ser humano.

La evaluación genera ese conocimiento a través de la medición, que es la que permite (como proceso) resumir informaciones que facilitan la comprensión de los fenómenos y etapas del desarrollo de niños y niñas, es este caso, costarricenses.

Sobre todo se busca evaluar la motricidad para conocer características, potencialidades y debilidades del desarrollo y hacer las intervenciones oportunas en los ciclos de transición y primaria.

Se resúmen 25 test, escalas o pruebas, en ellas se describen brevemente las habilidades a evaluar, edades y género, así como la referencia bibliográfica respectiva.

De esta forma el docente (de educación física o regular) cuenta con una guía que le va a posibilitar inicialmente y a corto plazo posibilidades de diagnóstico del desarrollo motor de sus alumnos y alumnas.

\author{
María de los Ángeles Monge Alvarado \\ Maureen Meneses Montero
}

Aceptado 9-IV-2002

\section{Introducción}

Desde sus comienzos, los estudios sobre la motricidad infantil se realizaron con la intención de conocer mejor a los sujetos y de poder establecer instrumentos para valorar, analizar y estudiar el status motor de estos (Ruíz, 1991).

A partir de los años 30 aparecen las escalas de desarrollo, donde el componente de la motricidad era el eje principal de la evaluación, a partir de entonces se generan lo que hoy se conoce como tests, exámenes, baterías o pruebas con la intención de evaluar, entre otras, las conductas motrices de las personas.

Es también a partir de este momento cuando grupos de profesionales, especialistas en diferentes áreas del saber se reúnen y toman decisiones y pautas importantes en el tema de la motricidad humana.

Entre los personajes más conocidos (Ruíz, 1991) encontramos a Gessell y sus escalas para valorar los diferentes aspectos del desarrollo del niño; Bayley, Shirley y McCarthy en cuyos exámenes psicológicos introducen el estudio de la motricidad infantil. Otra valoración importante fue la Escala de Desarrollo de Denver donde existe un apartado específico relacionado con la motricidad infantil.

La mayoría de estas escalas fueron realizadas para la primera infancia, como 
la Escala-Lezine de desarrollo psicomotor. Cabe destacar que se trabaja mucho en este período del desarrollo por las implicaciones afectivas, cognoscitivas, que tiene el movimiento y sus relaciones en la personalidad del ser humano.

\section{La evaluación}

La evaluación es el elemento crucial en la educación formal, en cualquiera de los diferentes ciclos, está en relación con los objetivos planteados.

Zamora (1998) la resume como "el juicio valorativo que se realiza a partir del establecimiento previo de los objetivos". Esta autora menciona dos funciones vistas desde el enfoque constructivista:

a. permite ajustar la ayuda pedagógica a las características y necesidades individuales de los alumnos, mediante aproximaciones sucesivas,

b. permite determinar el grado en que se ha conseguido las intenciones del proyecto curricular.

Para Bolaños (1986) son los procedimientos y criterios que se utilizan para saber sobre el logro de los objetivos propuestos. Además esta debe ser constante lo que servirá para hacer las rectificaciones en el proceso de enseñanza.

Mathieu (1999) se basa en Obrador y en Miras y Solé, que la determinan como la actividad con la que en la función de determinar criterios, se obtienen informaciones pertinentes sobre un fenómeno, una situación, un objeto o persona, se emite un juicio sobre el objeto de que se trate y adoptan una serie de decisiones relativas a éste.

Definida la evaluación se entiende la importancia que posee dentro del área de estudio. El valor de ella es relativo de los beneficios derivados del producto, siempre y cuando haya sido bien aplicada lo que contribuirá a mejorar el proceso y también a resolver las necesidades del producto.

Para realizar la evaluación es necesario seguir un procedimiento, Mathieu (1999) insiste que los datos por recolectar sean confiables y válidos. Los juicios por establecer según el valor de los datos van a responder a normas preestablecidas. Las decisiones por tomar se basan en la interpretación y juicios que se dan de los resultados de la medición.

\section{La medición}

La medición parte de la evaluación y su función es recolectar la información mediante la aplicación de diversos instrumentos como las listas de cotejo, escalas de clasificación, pruebas y otras.

La medición ha sido descrita por Zamora (1998) como el "proceso que permite resumir numéricamente las observaciones, respuestas, interacciones y registros de tal manera que se facilita su comprensión".

Al respecto Mathieu (1999) clarifica que la medición es una propiedad de las medidas precisas que dan por resultado datos, números con respecto a los individuos, objetos y situaciones medidas.

Shwanda (1978) operacionaliza todo el proceso como la evaluación parte de la educación, la medición es una fase de la evaluación y el instrumento a utilizar, es la herramienta de la medición.

Cuando se van a aplicar los instrumentos de medición se debe seguir con severidad ciertas condiciones, como las que Zamora (1998) señala:

a. las personas que lo aplican pueden variar las condiciones para los diferentes alumnos.

b. La calidad de los materiales que se suministran a los alumnos, la cantidad y la forma oportuna en que 
se presenten, también afectan los resultados.

c. Los ruidos, distorsiones para escuchar instrucciones, así como las interrupciones, deben ser eliminados.

d. La hora del día influye en la atención y la concentración.

e. Para que un test sea práctico debe ser fácil de administrar.

f Las instrucciones deben ser claras, concisas y precisas.

g. La facilidad está relacionada con el tiempo: si es pequeño el número de partes en las cuales debe de cronometrarse por separado, o si el tiempo no tiene importancia decisiva.

h. La edad de los evaluados debe considerarse tanto en el contenido como en el período de atención a éstos.

La selección de la prueba es un arte, es necesario conocer los criterios evaluativos, como la autenticidad científica, la posibilidad administrativa y su aplicación.

La autenticidad científica debe ser comprobada antes de su selección. Se debe estar seguro que la prueba fue diseñada científicamente y que mide precisamente lo que va a medir. Los criterios utilizados son la confiabilidad, objetividad y validez.

La confiabilidad y objetividad se refieren a la consistencia de la medición de la prueba. Shwanda (1978) ejemplifica ambos términos de la siguiente forma "la confiabilidad se refiere solo a un examinador repitiendo la misma prueba con el mismo grupo de sujetos y después comparando sus propios resultados mientras que la objetividad se refiere a dos o más examinadores comparando los resultados.

La validez se refiere a que si la prueba mide lo que pretende medir.
Si la prueba que se va a utilizar no tiene estos criterios es conveniente que el investigador los obtenga.

El segundo criterio es la posibilidad administrativa, esto quiere decir que debe atender a las posibilidades económicas y al tiempo requerido para ser administrado.

El tercer criterio es su aplicación, la cual atiende a los objetivos educacionales o de investigación que se tengan. Se deben tomar en cuenta las condiciones climáticas, de infraestructura y de equipo.

Mathieu (1999) es muy claro y estricto con respecto a la designación de una prueba y da algunas pautas prácticas que ha de tomarse en cuenta:

1. La prueba debe medir un aspecto importante.

2. Las pruebas deben seleccionarse de acuerdo a edad, sexo y de personas por medir.

3. Las pruebas deben discriminar en cuanto a destrezas.

4. Un requisito de la prueba es que pueda ser aplicada en forma colectiva, si fuera necesario.

5. Debe ser conocida y practicada previamente.

6. Debe ser sencilla como para que la pueda administrar una sola persona.

7. Si la prueba es dentro del tema de aptitud motriz, esta debe guardar la mayor similitud posible con la situación de juego.

8. Para la aplicación de la prueba se debe utilizar el mejor equipo humano y material.

9. La prueba debe ser fácil de preparar. 
10. Se deben orientar a todos los individuos que se van a medir.

11. Las pruebas deben tener normas establecidas que digan que es bueno, regular y malo.

12. Los resultados deben ser sencillos de interpretar y analizar.

13. La prueba debe medir un solo componente no varios a la vez.

14. La prueba debe dar seguridad a los participantes.

15. Las pruebas deben ser agradables a los ejecutantes.

\section{¿Para qué se evalúa el desarrollo motor?}

Es de suponer que el profesor o investigador realiza las mediciones de la motricidad para conocer a los sujetos, sus características, sus potencialidades, sus debilidades y así definir el perfil óptimo por alcanzar.

Las mediciones le van a permitir:

1. Conocer la situación actual del sujeto y su evolución a lo largo del tiempo.

2. Conocer los procesos que actúan en los sujetos y que determinan sus respuestas para poder decidir sobre esas situaciones.

3. Determinar qué hacer y recibir una retroalimentación informativa del efecto de la actuación pedagógica sobre los sujetos en cuestión.

4. Conocer situaciones motrices de acuerdo a la edad cronológica y en funcionamiento psicomotor adecuado (Ruíz, 1991).
Como educadores la evaluación es intrínseca a la labor que se desarrolla diariamente en el aula o gimnasio.

En el cuadro 1 Díaz (1993) expone un resumen que permite al educador ubicar las evaluaciones inicial, formativa y sumativa, con una escala de medición o instrumento adecuado al proceso.

\section{Procedimientos e instrumentos de evaluación y medición}

En Educación Física se puede hablar fundamentalmente de dos grandes tipos de evaluación: objetiva y subjetiva; y de dos técnicas de medición: cuantitativas y cualitativas.

La evaluación subjetiva depende directamente del criterio del profesor, aunque debe intentar que este sea el mismo al aplicarlo a los alumnos.

La evaluación objetiva es la que resulta de la utilización de pruebas o test ya establecidos y comprobados y que ya tienen la validez por utilizaciones anteriores. El profesor solo se tiene que limitar a aplicarlas.

La técnica cuantitativa pretende medir y cuantificar, de manera absoluta, un factor que se pretende observar en los alumnos, y que pueden ser aspectos como: tiempo, la distancia, el peso, el número de repeticiones, etc.

Por la técnica cualitativa se entiende aquella que se basa en una escala de medida mental dependiente del pensamiento del examinador. Por oposición a las técnicas cuantitativas, las técnicas cualitativas son generalmente asumidas como subjetivas, aunque es cierto que se puede conseguir una gran fiabilidad objetiva si se hace esta evaluación, por medio de técnicas de observación, estableciendo escalas categóricas de observación, procedimiento éste muy utilizado para trabajar en ciencias sociales (Díaz y otros, 1993).

Los instrumentos de evaluación siempre están acompañados de ayudas o 
Cuadro 1

RESUMEN EVALUACIONES INICIAL, FORMATIVA Y SUMATIVA.

\begin{tabular}{|c|c|c|c|}
\hline & $\begin{array}{c}\text { Evaluación } \\
\text { Inicial }\end{array}$ & $\begin{array}{l}\text { Evaluación } \\
\text { Formativa }\end{array}$ & $\begin{array}{l}\text { Evaluación } \\
\text { Sumativa }\end{array}$ \\
\hline & Pronóstico & Diagnóstico & Balance \\
\hline FUNCIONES & $\begin{array}{l}\text { Determinar la presencia o } \\
\text { ausencia de las habilidades pre- } \\
\text { viamente requeridas. } \\
\text { Determinar el nivel de dominio } \\
\text { previo. } \\
\text { Determinar las causas subya- } \\
\text { centes de reiteradas dificultades } \\
\text { en el aprendizaje. }\end{array}$ & $\begin{array}{l}\text { Información al alumno y } \\
\text { profesor sobre el proceso } \\
\text { de aprendizaje de la uni- } \\
\text { dad que se trate. } \\
\text { Para determinar: si los ob- } \\
\text { jetivos se van consiguien- } \\
\text { do y/o las posibles causas } \\
\text { de error en los elementos } \\
\text { del proceso. }\end{array}$ & $\begin{array}{l}\text { Valorar el aprendizaje una } \\
\text { vez que se ha realizado } \\
\text { para: determinar el nivel } \\
\text { alcanzado por el alumno } \\
\text { y/o determinar la eficacia } \\
\text { de todos los elementos del } \\
\text { proceso educativo. }\end{array}$ \\
\hline MOMENTO & $\begin{array}{l}\text { Antes de iniciar el aprendizaje. } \\
\text { Durante el aprendizaje, cuando } \\
\text { el alumno manifiesta repetidos } \\
\text { fracasos en el aprendizaje. }\end{array}$ & Durante el aprendizaje. & $\begin{array}{l}\text { Al final de un período más } \\
\text { o menos largo de instruc- } \\
\text { ción o al final del curso. }\end{array}$ \\
\hline $\begin{array}{l}\text { TIPO DE } \\
\text { INSTRUMENTO }\end{array}$ & $\begin{array}{l}\text { Pruebas de nivel. } \\
\text { Pruebas analíticas-diagnósticas. } \\
\text { Procedimientos de observación } \\
\text { directa o lista de control. }\end{array}$ & $\begin{array}{l}\text { Procedimientos de prueba } \\
\text { (objetivas o de ensayo) y } \\
\text { de observación, elaboradas } \\
\text { por el propio profesor. }\end{array}$ & $\begin{array}{l}\text { Pruebas de rendimiento } \\
\text { sumativas o finales. } \\
\text { Procedimientos de obser- } \\
\text { vación sobre el producto } \\
\text { más que sobre el proceso. }\end{array}$ \\
\hline DECISIONES & Reformar los objetivos & $\begin{array}{l}\text { Regulación del aprendizaje. } \\
\text { Adaptación de las activi- } \\
\text { dades de aprendizaje. }\end{array}$ & $\begin{array}{l}\text { Clasificación. } \\
\text { Certificar un nivel con- } \\
\text { seguido. }\end{array}$ \\
\hline
\end{tabular}

Tomado de: Díaz; García; Conte y otros (1993). Desarrollo Curricular para la Formación de Maestros Especialistas en Educación Física. España: Editorial Gymnos.

materiales anexos. Las listas de observación y control de las conductas perceptivomotrices (check list) son un ejemplo.

Cratty, McClenagham, y Gallahue o Williams, 1983 han elaborado algunos modelos (Ruiz, 1991), también Vayer (1977), ha agrupado un instrumento de gran valor, que mediante un golpe de vista sobre el trazado, permite advertir los puntos fuertes y débiles del sujeto en cuestión.

Da Fonseca (Ruíz, 1991) también ha elaborado un examen y batería psicomotriz en la que, sin resaltar tanto el factor cronológico, y siguiendo su modelo del funcionamiento psicomotor, ha seleccionado una serie de pruebas no importándole tanto lo que hace como el cómo lo hace.

Por último, se encuentran tests y baterías de tests que tienen su origen en el ámbito de la Educación Física y el Deporte: los tests de la AAPHER, el test de la coordinación corporal infantil de Kiphard (1976) y Schilling, la batería de Fleishman son ejemplos amplios y variados de diferentes instrumentos. 


\section{¿Qué criterios se utilizan para la selec- ción de un instrumento evaluativo?}

En muchas circunstancias la elección de un instrumento de medición se hace por lo general porque nos sentimos cómodos con él, por sus características intrínsecas o por la rutina de utilizarlo constantemente, sin cuestionar su auténtica realidad.

Es labor importante del profesional de la educación y de la motricidad el elegir un instrumento evaluativo con criterios serios y científicos. Se espera que al seleccionar un instrumento o test, tenga las siguientes características mínimas, que permitan en su aplicación garantizar los resultados:

1. Confiabilidad o la precisión con la que valora la estabilidad de los resultados si estos fueran requeridos en diferentes ocasiones. En otras palabras, la consistencia del instrumento.

2. Objetividad o independencia del instrumento del examinador.

3. Validez o mide lo que quiere medir.

4. Normas pautas para poder reflejar o comparar los resultados obtenidos en los sujetos.

5. Costo económico y de tiempo.

A todos éstos elementos deben sumarse el conocimiento del instrumento, presentación del mismo, y su capacidad para presentarlo, interpretarlo y elaborar el informe correspondiente, integrándolo con otra información obtenida.

Como se ha resumido hasta el momento, el instrumento de evaluación debe tener requisitos propios y además el evaluador debe estar claro en su objetivo y cómo pretende medir para hacerlo válido. En la educación física y el movimiento humano existen muchísimos test, escalas de valoración, baterías de pruebas, etc., todas concebidas como instrumentos de valoración motora general.

Se resumen a continuación varios test utilizados sobre todo en el nivel de ciclo de transición y primaria, los cuáles son de fácil uso y accesibles al personal docente.

\section{Test}

\section{Prueba patrones básicos de movimiento:}

Se aplica para edades entre los 3 y 6 años, determina la ejecución correcta de los patrones caminar, correr, saltar, brincar, patear, apañar, lanzar. Está estandarizado para ser aplicado en forma individual y en pequeños grupos. (Arce y Rivera, 1988; Cordero, 1988).

\section{Test de Pyfer:}

Prueba que se aplica de los 6 a los 14 años.

Determina si un niño puede ejecutar sus tareas básicas de desarrollo motor hasta una gran variedad de ellas.

Contiene un test básico que se aplica en forma grupal (5 niños), posterior y dependiendo de los resultados de está primera etapa se dan ítemes adicionales que se aplican en forma individual. Se permiten hasta 3 intentos por prueba. (Pyfer, 1990).

\section{Prueba KTK adaptada:}

Prueba validada en Costa Rica para niños de 5 1/2 a 8 1/2 años.

Se desarrolla a partir de la versión alemana, se cambia la manera de clasificar la ejecución del niño.

Tiempo de aplicación es de 10 a 15 minutos, valorando los aspectos:

- viga equilibrio,

- brincar obstáculos, 
- $\quad$ salto lateral,

- desplazamiento lateral,

- coordinación corporal.

Existe el manual de aplicación y las normas para Costa Rica. (Woodburn ${ }^{1}, 1991$ )

\section{Bruininks-Oseretsky}

Test de destrezas motora fina $\mathrm{y}$ gruesa.

Comprende 8 subtemas de 48 partes, conteniendo los siguientes aspectos:

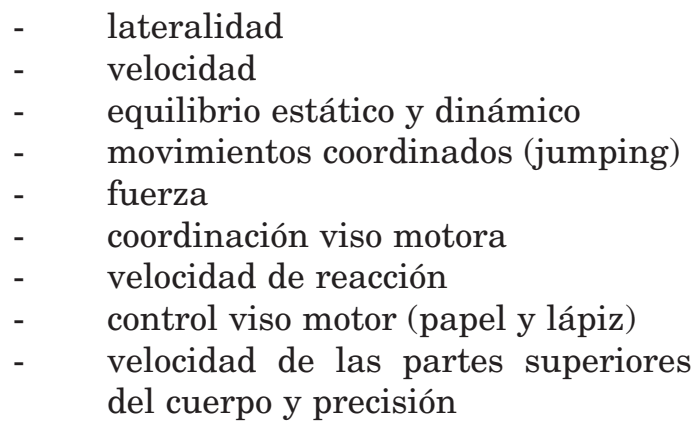

La prueba completa requiere un tiempo de 45 a 60 minutos por alumnos en la aplicación; existe además la posibilidad de seleccionar la llamada prueba corta que dura de 15 a 20 minutos por alumno.

Se establece como prueba longitudinal, lo que permite haber evaluado en un proceso educativo completo al niño escolar. (Bruininks, 1978).

\section{Prueba de desarrollo motor de la} U.N.A.

Basada en pruebas de Clark y Matawan, se hizo una adecuación ya que al traducir las pruebas no calzaron algunos términos. Valora a niños y niñas entre los $51 / 2$ a $81 / 2$ años. secciones:

Consta de 22 ítemes divididos en 6

- $\quad$ equilibrio

- movimientos asimétricos en espejo

\section{- $\quad$ orientación espacial \\ - destrezas locomotoras \\ - integración bilateral \\ - $\quad$ seguimiento ocular}

Tiempo de duración es de 10 y 15 minutos, se encuentra el manual y las normas de ponderación por edad y género. (Woodburn ${ }^{2}$, Rodríguez y Boschini, 1991).

\section{Prueba de diagnóstico de imagen corporal de la UNA}

Instrumento realizado como diagnóstico preliminar sobre conocimiento de la imagen corporal en niños y niñas de $51 / 2$ años a $81 / 2$ años (preescolar 1ro. y 2do. grado).

Se administra en forma individual, con una duración de 10 a 20 minutos. Consta de 94 ítemes, dividido en 4 subtest:

- planos corporales y partes cuerpo

- movimientos corporales

- lateralidad estática y dinámica

- direccionalidad estática y dinámica

Se encuentra el manual de aplicación y las normas. (Woodburn ${ }^{3}$ y Méndez, 1988).

\section{Prueba de sensibilidad motriz de Dayton}

Se aplica para niños y niñas de 4 y 5 años, los ítemes que se valoran son::

imagen corporal

espacio/direcciones

equilibrio

equilibrio/lateralidad

- lateralidad

- $\quad$ ritmo y control neuro-muscular

- integración derecho/izquierdo del cuerpo

- coordinación vista/pie

- control muscular finos

- percepción figura 
- discriminación auditiva

- coordinación vista/mano.

El profesor establece sus criterios de ponderación. (Richardson ${ }^{4}, 1986$ ).

\section{Prueba de la Escuela Meeting Street.}

Evalúa áreas motriz, viso motor y de lenguaje, a niños y niñas entre $51 / 2$ a 8 años.

El test mide la precisión con que el niño responde a estímulos visuales y auditivos a través de respuestas motoras y de lenguaje. Adaptada a vocabulario costarricense.

Se encuentra manual de aplicación y normas de referencia. (Woodburn ${ }^{5}, 1984$ ).

9. Test de desarrollo motor para niños preescolares de Clark

Evalúa niños y niñas entre 4 y 6 años pudiéndose aplicar tambien en primer grado. Los ítemes propuestos de aplicación son:

- $\quad$ equilibrio

- coordinación general fino/grueso

- $\quad$ espacio

- $\quad$ flexibilidad. (Richardson $\left.{ }^{6}, 1986\right)$.

\section{Escala de Desarrollo Integral (Edin)}

Evalúa el desarrollo integral del niño de 0 a 6 años, contiene ítemes de observación tanto del maestro como de los padres de familia.

Los ítemes que se observan entre 5 y 6 años son:

$\begin{array}{ll}- & \text { motora gruesa } \\ \text { - } & \text { motora fina } \\ \text { - } & \text { cognoscitiva } \\ \text { - } & \text { lenguaje } \\ \text { - } & \text { hocio ábitos de salud y nutrición. }\end{array}$

Permite al evaluador detectar problemas motores, neurológicos, etc. (Zamora, 1998).

\section{Prueba de auto concepto de Marti- nek-Zaichkowsky}

Consiste en un instrumento que no requiere habilidades de lectura, ni comunicación verbal, se establece para edades entre los 6 y 10 años, para niños y niñas.

Cada niño requiere de un folleto con las hojas que contienen los 25 ítemes del test. Se contesta en forma individual, la presentación de las preguntas por contestar del evaluado son dos acciones opuestas en forma de dibujo, el niño o niña debe escoger con cuál se identifica más. Se aplica en grupos de hasta 30 niños, el manual establece el número de asistentes de la prueba según el número de evaluados, se requiere de 10 a 15 minutos en su administración, sin embargo si un niño requiere de más tiempo se le debe conceder.

La escala del test va de 0 puntos hasta 25 puntos como máximo, se encuentra estandarizado para Costa Rica. (Martinek y Zaichkowsky, 1977; Richardson ${ }^{7}, 1990$; Meneses y Monge, 1997).

\section{Test de imitación de gestos de Berges-Lezine}

Este test requiere de la imitación de uno o varios gestos propuestos por el examinador y que supone el conocimiento y dominio del cuerpo como instrumento y la posibilidad de utilizarlo con un fin de acuerdo a un modelo.

El evaluador presenta modelos representativos como triángulos, círculos, líneas rectas, líneas diagonales, etc., y el evaluado las representa con sus manos o cuerpo, hace una imitación de las mismas. (Berges y Lezine, 1975).

\section{Test de dominancia lateral de Schilling (TDL)}

Este test tiene la finalidad de valorar el rendimiento lateral del niño mediante una prueba de punteado de 150 circulitos 
con la ayuda de un lapicero especial, uno detrás de otro, una vez con cada mano. Estos círculos están ordenados de tal forma que conforman un muñeco. El muñeco sostiene dos pelotas que sirven como prueba inicial.

Como parámetros de evaluación utiliza el tiempo de realización y los errores cometidos, con una interpretación directa o traducibles en índice de lateralidad. (Schilling, 1974).

\section{Test del pato y el conejo de Peret}

Esta prueba está elaborada para detectar la dominancia ocular. Consiste en que el examinador presenta y pregunta sobre la figura a 60 centímetros de distancia, de tal manera de que si el niño ve un conejo denota zurdera ocular, y si lo que ve es un pato es ocularmente diestro. (Ruíz, 1991).

\section{Prueba para determinar la lateralidad}

Antonio Alayón, neurólogo, propone esta prueba para determinar la lateralidad física y sensorial de los niños y las niñas como base de una "estimulación precoz". Tomando en cuenta los períodos sensitivos de los infantes de 0 a 6 años y la lateralidad como la última de las etapas del desarrollo psicomotor. (Alayón, 2001).

\section{Escala Gessell de desarrollo infantil}

Esta escala fue elaborada por Gessell tras el estudio minucioso de la conducta del niño y abarca 4 áreas: adaptiva, verbal, motriz y social. Está elaborada para estudiar a los niños hasta los 6 años. Esta escala ha sido muy utilizada por diversos especialistas del desarrollo infantil aunque ha sido criticada de excesivamente normalizante. $\left(\right.$ Gessell $^{8}$, 1980; Gessell ${ }^{9}$ y Amatruda, 1981).

\section{Escala Brunet-Lezine de Desarrollo Motor}

Brunet y Lezine entre 1978 y 1980, a partir de los trabajos de Gesell elaboraron una escala para el estudio del desarrollo psicológico del niño donde las situaciones motrices son numerosas. Las pruebas se componen de situaciones que se proponen al niño verbal y no verbalmente para observar su desempeño.

Los test están divididos en dos apartados uno más simple y otro más complejo, y abarca a los 6 primeros años.

Todas estas escalas tanto la de Gessell, como la de Brunet-Lezine, tienen como misión determinar el cociente de desarrollo del niño al comparar la edad cronológica real con la edad del desarrollo obtenida de la aplicación de las diversas pruebas. (Brunet y Lezine, 1980).

\section{Lista de control de conductas perceptivo-motrices de Cratty}

Consiste en una lista de control de conductas perceptivo motrices que recoge de forma escueta las característica que se consideran más resaltables del comportamiento perceptivo motor de los niños.

Según el autor se puede esperar que el $75 \%$ de los sujetos en las edades indicadas, que van de los 2 a los 8 1/2 años, las ejecuten sin dificultad.

Asimismo a lo largo de las edades se van especificando, las posibles diferencias individuales. $\left(\right.$ Cratty $^{10}, 1979$; Cratty ${ }^{11}$, 1967).

\section{Test de diagnóstico de la capacidad motriz de Arheim-Sinclair}

Resume otros ítemes de diferentes test, los cuáles fueron seleccionados para servir de instrumento en la detección de los sujetos con necesidad de atención especial a su motricidad. Los autores elaboran una normalización de los mismos y lo presentaron en el texto "El niño torpe" en el año 1976. 
Los ítemes que componen esta batería son:

- enhebrado de cuentas,

- $\quad$ rapidez de golpeo (tapping),

- salto horizontal,

- lanzamientos de precisión,

- flexibilidad,

- cambios de posición (agilidad),

- carrera de agilidad,

- $\quad$ flexiones de brazos. (Ruíz, 1991).

20. Test de coordinación corporal infantil (Hamm-Marburg Der Körper koordinations-test für Kinder)

Este es un test ideado para detectar los problemas de coordinación corporal que pueden manifestarse en los niños de 5 a 14 años. Después de una serie de estudios, las pruebas iniciales fueron reducidas a 4 :

- marcha hacia atrás sobre barras de equilibrio de diferentes anchuras (6, 4, 5, y $3 \mathrm{~cm}$.),

- $\quad$ saltos sobre bloques de goma espuma con una pierna sobre alturas crecientes, - desplazamientos sobre soportes (20”),

- $\quad$ saltos laterales sobre una línea en el suelo (15”).

Los parámetros evaluados son tiempo, suma de errores, amplitud y precisión. Cada tarea está afectada por la existencia de un Cociente motor específico y se acaba con un Cociente Motor Global. Tiene como inconveniente el que solo requiere una parte muy concreta de tareas de coordinación. (Kiphard y Schilling, 1976).

\section{Batería básica de aptitud física de Fleishman}

Es una batería destinada a adolescentes entre los 12 y 18 años, no se aplica para edades infantiles, y en su forma básica está compuesta de 10 test obligatorios y 4 optativos.
Los factores estudiados y evaluados son:

- flexibilidad estática y dinámica,

- agilidad,

- fuerza explosiva de los miembros superiores,

- fuerza de agarre. Dinamometría manual,

- fuerza de tracción en barra,

- fuerza abdominal,

- $\quad$ agilidad. Salto del cable,

- $\quad$ endurecimiento. 600 yardas,

- equilibrio estático,

- $\quad$ velocidad de carrera 50 yardas,

- potencia extensora de piernas. Salto horizontal. (Fleischman, 1964).

\section{Examen psicomotor de Vayer}

Vayer, en los años 70, publicó como resultados de sus experiencias e investigaciones con los niños con necesidades especiales e inadaptados, un examen psicomotor para la primera y segunda infancia fruto de la unión de pruebas y tests de diferente origen, pero con la finalidad de obtener una información adecuada del niño (Ruíz, 1991).

Vayer realizó la construcción del examen utilizando tests de Ozeretsky revisados por Guilmain, con dos modelos: 1) 25 años; 2) 6-12 años. Esta conformado por pruebas de la escala de Brunet-Lezine, pruebas de evaluación intelectual de Terman, Merrill y Binet Simon, revisadas por Terman y pruebas de imitación de estos de Berges-Lezine.

El examen psicomotor abarca aspectos del comportamiento psicomotor tales como la coordinación óculo manual, coordinación dinámica, control del propio cuerpo, organización perceptiva, observaciones de la lateralidad, estructuración espaciotemporal y conducta respiratoria.

Los resultados de las diversas pruebas son plasmados en un perfil que manifiesta el estado del sujeto con respecto a su 
edad, los puntos fuertes y los puntos débiles. Se puede considerar uno de los instrumentos más utilizados en el ámbito educativo. (Ruíz, 1991; Vayer, 1980).

\section{Observación psicomotora de Da Fonseca}

Da Fonseca elaboró una batería de observación psicomotriz basada en sus estudios sobre el funcionamiento psicomotor del niño y la teoría Luriana de los niveles funcionales del cerebro, trata de captar la personalidad psicomotriz del niño, su estilo psicomotor. Para este autor el acto motor debe considerarse como un elemento del conjunto de operaciones cognitivas que son llevadas a cabo por el niño.

La batería está elaborada para niños de 4 a 14 años y basada en 7 áreas de observación:

1. Tonicidad

2. Equilibración

3. Lateralización

4. Noción del cuerpo

5. Estructuración espacio temporal

6. Praxia global

7. Praxia fina

La escala de puntuación es de tipo cualitativo a diferencia de otras baterías.

La escala va de 1 a 4 . El 1 correspondería al sujeto apráxico, incapaz de llevar a cabo la tarea sugerida (insuficiente). El 2 al sujeto dispráxico, aquel que primero manifiesta dificultades de control. Al 3 la realización controlada y adecuada. Y al 4 la realización perfecta, económica, armoniosa y bien controlada.

La dificultad de esta forma de evaluar está en el dominio del conocimiento del examinador de las diversas pruebas, de lo que se puede esperar en el niño normal en ellas y en saber observar detenidamente todo lo que ocurre en el período de examen. (Fonseca da, 1996).

\section{Evaluación del Perfil Psicomotor}

Johanne Durivage (1987) presenta esta prueba que permite determinar el nivel de desarrollo de cada niño, comprobar las adquisiciones hechas y detectar las dificultades o retrasos.

Este instrumento está diseñado con tres columnas: objetivos, actividades que se van a realizar y una lista de hechos observables al ejecutar las actividades y se anota sí o no.

Por último se encuentra una hoja de valoración grupal por medio de la cual el maestro o maestra extraerá el perfil psicomotor del grupo. (Durivage, 1987).

\section{Test para determinar el predominio lateral}

Sirve para determinar el predominio lateral en los miembros superiores e inferiores de cada sujeto, el mismo se realiza ejecutando diferentes tareas motrices. El evaluador asigna la puntuación correspondiente, de acuerdo a sus criterios. (Le Boulch, 2001).

\section{Conclusión}

El educador físico debe colaborar con el desarrollo integral del individuo, para que su capacidad potencial sea utilizada al máximo, y es aquí donde una buena evaluación en las conductas motoras (principalmente), afectivas, sociales y cognitivas va a permitir el desarrollo de actitudes y aptitudes globales.

Es importante la concepción de la función que ejerce el docente como formador y guía en los períodos "críticos" del desarrollo sobre todo en niños y niñas de edad preescolar y escolar.

La evaluación definitivamente permite y proporciona información valiosa que orienta al docente en el proceso de enseñanza aprendizaje. 


\section{Notas}

1. Woodburn, S.; Méndez,C.; Boschini, C. Prueba KTK Adaptada: Prueba de Coordinación Corporal para Niños. Heredia: Editorial EUNA, 1991.

2. Woodburn, S.; Rodríguez, F.; Boschini, C. Prueba de Desarrollo Motor (PDM-UNA). Heredia: Editorial EUNA, 1991.

3. Woodburn, S.; Méndez, C. Prueba para el Diagnóstico de la Imagen Corporal. Heredia: Editorial EUNA. 1988.

4. Richardson, M.J. Medición de la Sensibilidad Motriz de Dayton para Niños de 4 a 5 Años. San Pedro: Universidad de Costa Rica. (Folleto mimeografiado) 1986 .

5. Woodburn, S. El Diagnóstico Temprano de Problemas de Aprendizaje: Test de la Escuela Meeting Street Adaptado. Heredia: Editorial EUNA. 1984.

6. Richardson, M.J. Test de Desarrollo Motor para Niños Preescolares de Clark. San Pedro: Universidad de Costa Rica. (Folleto mimeografiado) 1986.

7. Richardson, M.J. "Self Concepts of Costa Rican Elementary School Children”. Perceptual and Motor Skills, 1990, 70, 1331-1334.

8. Gessell, A. Gessell Developmental Test Materials. INC. Houston. 1980.

9. Gessell, A.; Amatruda, C. Diagnóstico del Desarrollo Normal y Anormal del Niño. Buenos Aires: Paidós. 1981.

10. Cratty, B Motricidad y Psiquismo en Educación y Deporte. España: Miñón. 1979.

11. Cratty, B. Developmental Sequences of Perceptual-Motor Task. New York: Educational Activities. 1967.

12. Vayer, P. El Equilibrio Corporal. Barcelona: Científico-Médica. 1980.

\section{Referencias bibliográficas}

Alayón, A. Estimulación Temprana y Maduración. 2001.
Disponible en: http://www.edufam. net/escpad/temtrab275.htm

Arce, R.; Rivera, J. Estandarización de una Batería de Escalas de Clasificación de Patrones Básicos Locomotores y Manipulativos para Niños Costarricenses de Edad Preescolar. Universidad de Costa Rica. Tesis de grado, 1988.

Besges, J.; Lezine, I. Test de Imitación de Gestos. Barcelona: Científico- Médica. 1975.

Bolaños, G. Educación Física y Expresión Corporal. San José: Universidad Estatal a Distancia. 1986.

Bruininks, R. Bruininks-Oseretsky Test of Motor Proficiency. Circle Pines, Minnesota: American Guidance Service, 1978.

Brunet, O.; Lezine, I. El Desarrollo Psicológico de la Primera Infancia. Madrid: Pablo del Río. 1980.

Cordero, R. Normas de Comportamiento Motor en los Niños Preescolares. Universidad de Costa Rica. Tesis de grado, 1988.

Cratty, B. Motricidad y Psiquismo en Educación y Deporte. España: Miñón. 1979.

evelopmental Sequences of Perceptual-Motor Task. New York: Educational Activities. 1967.

Díaz, García y otros Desarrollo Curricular para la Formación de Maestros Especialistas en Educación Física. España: Editorial Gymnos. 1993.

Durivage, J. Educación y Psicomotricidad. Manual para el Nivel Preescolar. México: Trillas. 1987. 
Fleishman, E. The Structure and Measurement of Physical Fitness. New Jersey: Prentice-Hall. 1964.

Fonseca da, V. Estudio y Génesis de la Psicomotricidad. Barcelona: INDE Publicaciones. 1996.

Gessell, A. Gessell Developmental Test Materials. INC. Houston. 1980.

Amatruda, C. Diagnóstico del Desarrollo Normal y Anormal del Niño. Buenos Aires: Paidós. 1981.

Kiphard, E. Problemas de Movimiento y Coordinación en la Escuela Primaria. Buenos Aires: Kapelusz. 1976.

Kiphard, E.; Schilling, F. "The Body Coordination Test". JOPERD 47(37). 1976.

Le Boulch, J. Test para Determinar el Predominio Lateral. 2001.

Disponible en: www.xtec.es/ jisanz/avalua/leboulch.html

Martinek, T.J.; Zaichkowsky; L.D. Manual for the Martinek-Zaichkowsky Self Concept Scale for Children. St. Louis, MO: Psychologists \& Educators. 1977.

Mathieu, W. Medición y Evaluación de la Educación Física. Universidad de Costa Rica 1999. (Folleto mimeografiado).

Meneses, M.; Monge, M. "Implementación del Test de Martinek- Zaichkowsky de Autoconcepto, a Grupos de Niños y Niñas de 6, 7, 8, 9 y 10 Años del Instituto Franco Costarricense". Revista Educación, 21 (2): 65-72, 1997.

Pyfer, J. Valorando el Desarrollo Motor. Taller presentado en la VIII Conferencia Interamericana de Educación
Física, Recreación y Deporte para Personas Discapacitadas. Universidad de Costa Rica, 1990.

Richardson, M.J. Medición de la Sensibilidad Motriz de Dayton para Niños de 4 a 5 Años. San Pedro: Universidad de Costa Rica. (Folleto mimeografiado) 1986.

- Test de Desarrollo Motor para Niños Preescolares de Clark. San Pedro: Universidad de Costa Rica. (Folleto mimeografiado) 1986.

. "Self Concepts of Costa Rican Elementary School Children". Perceptual and Motor Skills, 1990, 70, 1331-1334.

Ruíz. L. Desarrollo Motor y Actividades Físicas. España: Gymnos. 1991.

Schilling, F. Köperkoordinationtest für Kinder KTK. Weinheim Beltztest GMBH. 1974.

Shwanda, N. Medición y Evaluación en la Educación Física..Escuela Educación Física y Deportes. Universidad de Costa Rica. (Folleto mimeografiado) 1978 .

Vayer, P. El Niño Frente al Mundo. Barcelona: Científico-Médica. 1977.

El Equilibrio Corporal. Barcelona: Científico-Médica. 1980.

Williams, H. Perceptual and Motor Development. New Jersey: Prentice Hall. 1983.

Woodburn, S.; Méndez,C.; Boschini, C. Prueba KTK Adaptada: Prueba de Coordinación Corporal para $\mathrm{Ni}$ ños. Heredia: Editorial EUNA, 1991. 
Woodburn, S.; Rodríguez, F.; Boschini, C. Prueba de Desarrollo Motor (PDMUNA). Heredia: Editorial EUNA, 1991.

Woodburn, S.; Méndez, C. Prueba para el Diagnóstico de la Imagen Corporal. Heredia: Editorial EUNA. 1988.
Woodburn, S. El Diagnóstico Temprano de Problemas de Aprendizaje: Test de la Escuela Meeting Street Adaptado. Heredia: Editorial EUNA. 1984.

Zamora, D. La Evaluación del Niño y la Niña en Preescolar. Costa Rica: Universidad Estatal a Distancia. 1998.

Ma. de los Ángeles Monge Alvarado Profesora Asociada de la Escuela de Educación Física y Deportes de la Universidad de Costa Rica

Maureen Meneses Montero Profesora Catedrática de la Escuela de Educación Física y Deportes de la Universidad de Costa Rica 